\title{
DRIVING WITH PARA-CENTRAL VISUAL FIELD LOSS: PILOT STUDY
}

\author{
Matt Bronstad, Alex Bowers, Amanda Albu, Robert Goldstein, \& Eli Peli \\ Schepens Eye Research Institute, Department of Ophthalmology, Harvard Medical School \\ Boston, Massachusetts, USA \\ Email: matthew.bronstad@schepens.harvard.edu
}

\begin{abstract}
Summary: We studied how para-central visual field loss affects pedestrian detection in a driving simulator. Participants with para-central field loss had relatively good visual acuity $(20 / 15-20 / 60)$ and 3 of 5 met local vision requirements for an unrestricted drivers license; however, they had lower detection rates and longer reaction times to pedestrians likely to appear within the blind area than in their seeing areas. They were at collision risk for $7 \%$ to $30 \%$ of pedestrians, whereas controls were at a collision risk for 0 to $4 \%$ of pedestrians.
\end{abstract}

\section{INTRODUCTION}

Driver licensing vision requirements in the United States consider visual acuity, and many states measure peripheral visual field loss (Peli \& Peli, 2002). However, central field loss (CFL) is rarely considered. Some countries, such as the U.K. (Rauscher et al., 2007) and Canada (YazdanAshoori \& ten Hove, 2010) have regulations limiting licensing with central visual field loss, but little or no data supports these regulations.

We are evaluating how scotomas (blind areas) in the central visual field impact driving in a simulator, and previously reported the effects of CFL resulting from foveal damage (Bronstad $e t$ al., 2009). There are many visual conditions, however, that involve para-central field loss (pCFL) - visual loss that affects the central field (central $60^{\circ}$ diameter of the visual field) but with an intact fovea. This can result from age-related macular degeneration with foveal sparing; post-chiasmal optic tract lesions causing partial hemianopia; or monocular blindness, where the physiological blind spot of the fellow eye is not compensated. In pCFL there may be normal peripheral vision, and often, normal visual acuity (Petzold \& Plant, 2005); thus, people with pCFL may drive legally in the U.S.A. The impact of the para-central scotomas on driving, particularly hazard detection, is unknown.

We present a case series demonstrating that pCFL affects detection of potential collision hazards (pedestrians). We hypothesized that participants' scotomas decrease detection rates and increase reaction times to pedestrians at locations likely to be obscured by each participant's scotoma. These predictions assume that participants looked straight down the road most of the time when driving. Such behavior is exhibited even by experienced drivers (Underwood, 2007). Scanning eye movements could compensate for visual field loss; however, many people with CFL are unaware of their field loss (Fletcher, 2011), and might therefore not compensate by scanning. Furthermore, data from studies of eye movement behaviors of people with visual field loss during naturalistic tasks (e.g., walking) suggest little or no compensatory scanning (Hassan, Geruschat, \& Turano, 2005; Turano, Geruschat, \& Baker, 2002; Vargas-Martín \& Peli, 2006). However, if participants did scan, we still expected longer reaction times for pedestrians 
appearing at scotoma locations as they would likely remain in the scotoma until scanning brought them out of the scotoma.

\section{METHODS}

\section{Participants}

Five participants with binocular pCFL; two with age-related macular degeneration (AMD), and three with partial hemianopia (without spatial neglect) participated. The extent and location of the scotomas varied (Figure 1). Participants A and B had partial left hemianopia that could delay detection of pedestrians who appeared in the left visual field (difficulty at $-14^{\circ}$ and $-4^{\circ}$ ), while participant $\mathrm{C}$ had a right upper quadranopia (partial hemianopia) that might delay responses on the right (difficulty at $4^{\circ}$ and $14^{\circ}$ ). Participant $\mathrm{D}$ had no visual loss in his right eye, but his left eye had a large scotoma in the right side of the visual field that overlapped with the physiological blind spot (the normal blind spot where the optic nerve leaves the eye) in his right eye (difficulty at $14^{\circ}$ ). Participant E had a "ring scotoma" due to AMD leaving him with a small area of residual central vision (diameter $\sim 8^{\circ}$ ) surrounded by a large scotoma that might delay target detection on both sides (difficulty at $-14^{\circ}, 4^{\circ}$ and $14^{\circ}$ ).
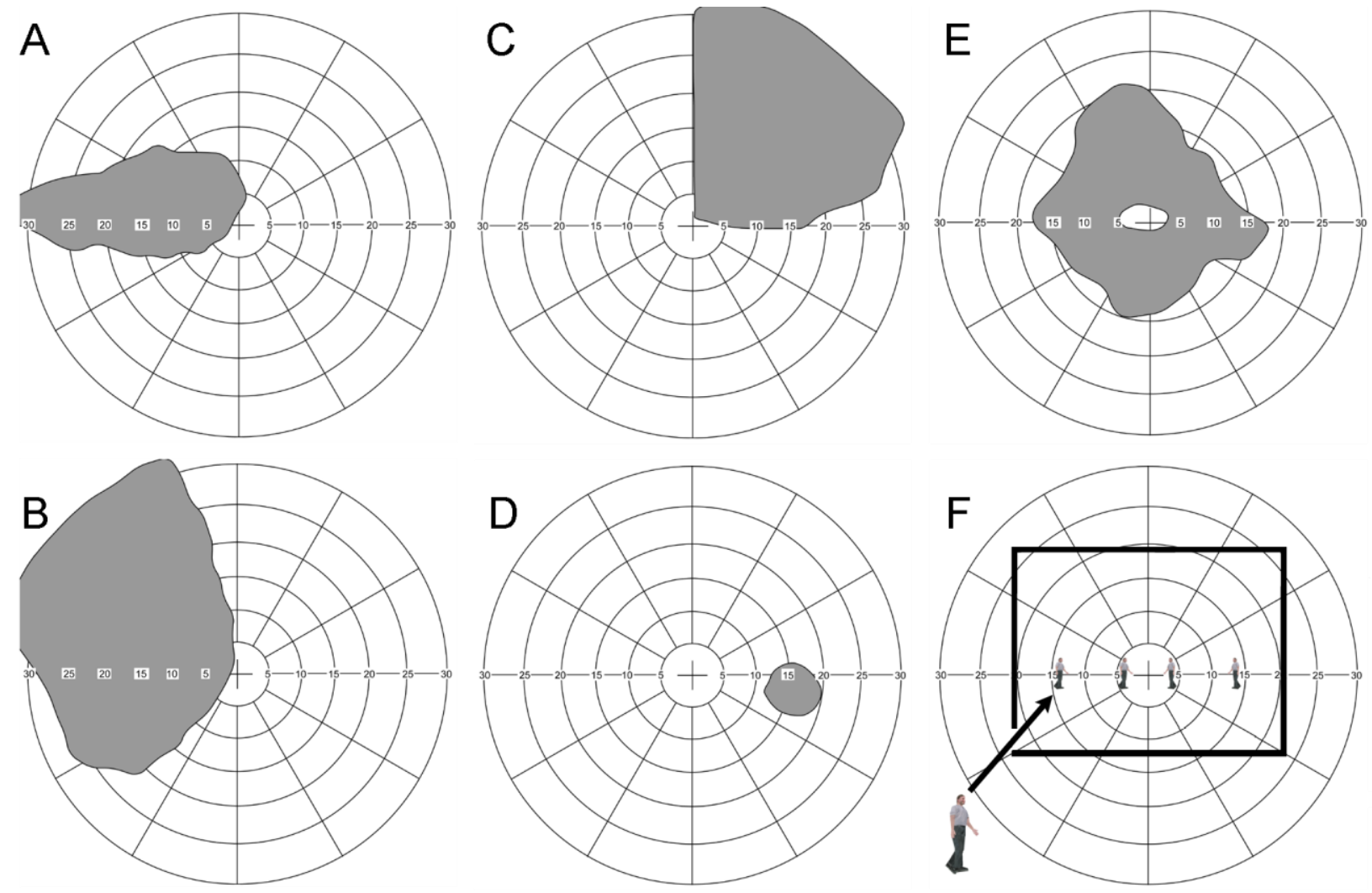

Figure 1. Binocular visual field plots for the 5 participants with pCFL; each plot covers $60^{\circ}$ (diameter) of central visual field; F: approximate locations and size of the pedestrians at $3 \mathrm{~s}$ after appearance (thick black lines represent the extent of the central monitor, which has a slight vertical asymmetry) 
Acuity and peripheral vision of 3 of the pCFL participants was sufficient for unrestricted licensing in Massachusetts, and all had driven recently (one stopped in 2002, but drove his wife for medical treatments for a few months a short time before participating). All subjects were matched with normally-sighted controls for age, sex and number of years driving.

\section{Procedure}

Participants underwent cognitive testing (Short Portable Mental Status Questionnaire; Pfeiffer, 1975) and vision screening, including visual acuity and Goldmann peripheral field measurement. Size and position of the paracentral scotoma was measured under binocular viewing using kinetic perimetry $\left(12 \mathrm{~mm}, 0.7^{\circ}\right.$ visual angle, bright target, $97 \mathrm{~cd} / \mathrm{m}^{2}$ viewed at $1 \mathrm{~m}$ over a $23 \mathrm{~cd} / \mathrm{m}^{2}$ grey background) with a custom-designed computerized system (Woods, Apfelbaum \& Peli, 2010). Participants then completed two 2-3 hour simulator driving sessions.

The simulator is a FAAC PP- 1000 with $225^{\circ}$ horizontal field of view, a motion seat, and controls typical for an automatic transmission car. After acclimation to the simulator, participants completed 3 city $(30 \mathrm{mph})$ and 2 rural, undivided highway $(60 \mathrm{mph})$ drives in each session (Bowers et al., 2009; Bronstad et al., 2009). Drives (each 10-12 min.) had traffic and required various maneuvers (right turns, left turns etc.).

Table 1. Visual demographics of the participants

\begin{tabular}{|c|c|c|c|c|c|c|}
\hline & YOB - Sex & Diagnosis & Side of Loss & Visual acuity (OU) & Driving years \\
\hline & A & $1933-\mathrm{F}$ & Hemianopia & Left & $20 / 30$ & 1954-present \\
\hline & B & $1949-\mathrm{M}$ & Hemianopia & Left & $20 / 15$ & 1965-present \\
\hline & $\mathrm{C}$ & $1972-\mathrm{M}$ & Hemianopia & Right & $20 / 20$ & 1987-present \\
\hline & D & $1934-\mathrm{M}$ & AMD* & Right & $20 / 25$ & 1950-present \\
\hline & $\mathrm{E}$ & $1931-\mathrm{M}$ & AMD & "Ring" & $20 / 60$ & $1947-2002$ \\
\hline Mat & ed to $\mathrm{A}$ & $1931-\mathrm{F}$ & $\mathrm{n} / \mathrm{a}$ & $\mathrm{n} / \mathrm{a}$ & $20 / 25$ & 1947-present \\
\hline “ & “B & $1951-\mathrm{M}$ & $\mathrm{n} / \mathrm{a}$ & $\mathrm{n} / \mathrm{a}$ & $20 / 25$ & 1970-present \\
\hline “ & " $\mathrm{C}$ & $1964-\mathrm{M}$ & $\mathrm{n} / \mathrm{a}$ & $\mathrm{n} / \mathrm{a}$ & $20 / 20$ & 1981-present \\
\hline “ & “D & $1937-\mathrm{M}$ & $\mathrm{n} / \mathrm{a}$ & $\mathrm{n} / \mathrm{a}$ & $20 / 15$ & 1951-present \\
\hline “ & “E & $1931-\mathrm{M}$ & $\mathrm{n} / \mathrm{a}$ & $\mathrm{n} / \mathrm{a}$ & $20 / 25$ & 1949-present \\
\hline
\end{tabular}

*AMD with large CFL in left eye, and physiological scotoma in the right visual field (Figure 1d)

Participants were asked to follow all of the normal rules of the road, follow directions played from pre-recorded audio files, and to press the horn as soon as they saw the pedestrian which appeared at one of four positions relative to the participant's car heading $\left(-14^{\circ},-4^{\circ}, 4^{\circ}\right.$, and $14^{\circ}$; Figure 1F). We gave no instructions about eye or head scanning. There were 12 pedestrian appearances in each drive (120 across the two simulator sessions). In city drives the life-sized pedestrians appeared 220ft from the participant's vehicle, 5 seconds distant at the posted $30 \mathrm{mph}$ speed. Pedestrians walked or ran (exhibiting biological motion) toward the participant's lane on a collision course with the vehicle, maintaining a relatively stable eccentricity with respect to the vehicle heading (constant bearing angle; Cherdenon et al., 2002) and consequently presenting a realistic hazard. Thus, if participants looked straight down the road most of the time, the pedestrians would remain in approximately the same position in the participant's visual field. However, to avoid actual collisions, the pedestrian figures were programmed to stop just before they reached the participant's travel lane. 
Pedestrians' motion was scripted on the assumption that the participant's vehicle traveled at the posted speed limit; however, they were designed to be robust for speeds within $\pm 10 \mathrm{mph}$ of the limit as we expected that participants with pCFL might drive more slowly. Primary outcomes were detection rates and reaction times.

\section{Analyses}

As detailed above, we expected longer reaction times for pedestrians that were likely to appear within scotoma locations, and made specific predictions for each pCFL participant (Figure 1). Reaction times at "scotoma" and "non-scotoma" locations were compared on an individual basis for each pCFL participant. In addition, reaction times at scotoma and non-scotoma locations were compared to the matched controls at the corresponding locations. As reaction times were not normally distributed, Kruskall Wallis tests were used for within-subjects comparisons and Mann-Whitney U tests were used for between group comparisons. Data were pooled across city and highway drives.

We also calculated whether for each pedestrian appearance there might have been a potential collision. That is, if pedestrians continued walking into the travel lane (which they did not), participants would have collided with them and/or had to steer around them. Potential collisions included both detection failures as well as situations in which response times were so long that there would not have been sufficient time (distance) to complete a braking maneuver (given participant's speed at time of honk and assuming deceleration of $5 \mathrm{~m} / \mathrm{s}^{2}$ immediately after honk; Evans, 2004).

\section{RESULTS}

Controls detected all pedestrians, whereas participants with pCFL detected $100 \%$ of pedestrians that likely appeared in their seeing areas but only $92.5 \%$ of pedestrians that likely appeared in their scotomas (Figure 2). In comparison to their reaction times at non-scotoma locations, the reaction times for pCFL participants for pedestrians at scotoma locations were delayed, $\chi^{2}=$ $7.21, p=0.007$. However at non-scotoma locations, reaction times were only slightly elevated relative to controls (the effect was not significant, $Z=1.54, p=.13$ ).

Projected collisions were higher at scotoma than non-scotoma locations for pCFL participants $\left(\chi^{2}=9.98, p<.01\right)$, with rates from $0-12 \%($ median=2\%) for non-scotoma locations and $8-47 \%$ (median $=30 \%$ ) for scotoma locations. pCFL participants were at collision risk for $7 \%$ to $30 \%$ $($ median $=10 \%)$ of all pedestrian appearances. Controls were at collision risk for $0-4 \%$ (median $=2 \%$ ) of all pedestrian appearances.

Left scotomas. Both participants showed reduced detection rates and longer reaction times to pedestrians on the left (Figure 2). As predicted, the $-4^{\circ}$ and $-14^{\circ}$ locations were delayed compared to $4^{\circ}$ and $14^{\circ}$ for both participants $\left(\chi^{2}=49.12\right.$ and 18.66 , respectively, both $\left.p s<0.001\right)$, but participant $\mathrm{B}$ was only slightly longer to the $-4^{\circ}$ targets.

Right scotomas. Participant C (Figure 2) demonstrated reduced detection at $4^{\circ}(95 \%)$, even more markedly reduced detection at the $14^{\circ}$ location $(80 \%)$, and elevated reaction times to targets at $4^{\circ}$ 
and $14^{\circ}\left(\chi^{2}=27.36, p<0.01\right)$. Participant $\mathrm{D}$, with a much smaller scotoma, detected all targets and had only slightly longer reaction times at the $14^{\circ}$ location $\left(\chi^{2}=8.03, p<0.01\right)$.

Ring scotoma. Participant E (Figure 2) had deficits at $-14^{\circ}, 4^{\circ}$ and $14^{\circ}$, as predicted given his large pCFL and had elevated reaction times to pedestrians at these locations $\left(\chi^{2}=20.00\right.$, $p<0.001)$.
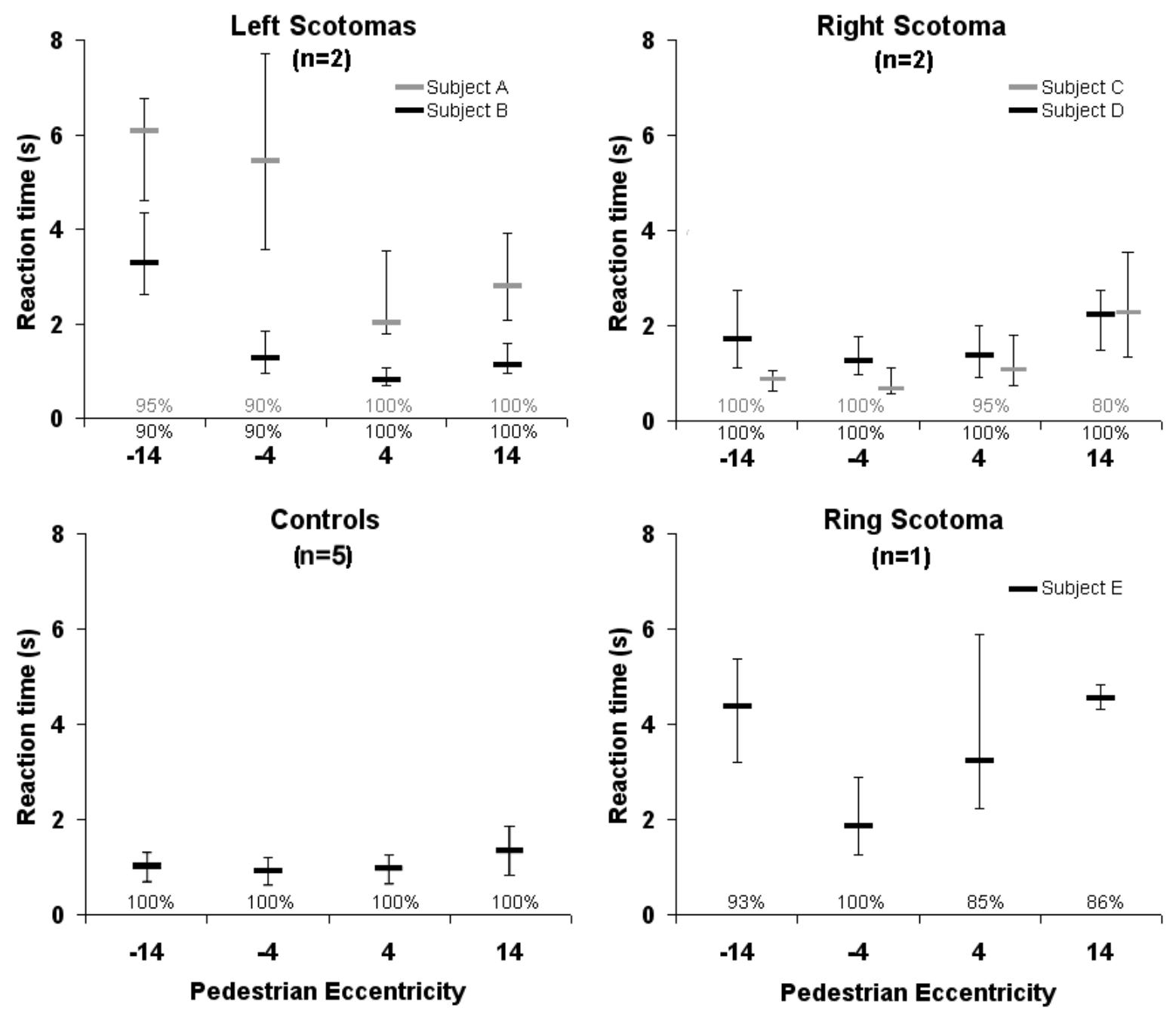

Figure 2. Reaction times to the pedestrian targets; bars indicate reaction time medians; whiskers show $25 \%$ and $75 \%$ quartiles; data are pooled for the 5 controls (percentages next to the $\mathrm{x}$-axis are detection rates)

\section{DISCUSSION}

Our results replicate the finding that central scotomas increase reaction times in visual field areas corresponding to the scotoma location/side (Bronstad et al., 2009), and are in good agreement with our predictions. The large scotomas (from partial hemianopia and AMD) more strongly impacted detection rates and reaction times than the small physiological blind spot (small 
increased latency at $+14^{\circ}$ ). This is encouraging for drivers with monocular vision with a physiological blind spot scotoma, but needs further verification.

Extrapolating to a real world situation, between 0 and $10 \%$ of pedestrians were not detected at all by participants with pCFL. If an undetected pedestrian had continued walking towards the participants' vehicle, there would have been a collision. In addition, long reaction times meant that participants may not have been able to stop in time to prevent some additional collisions. Analyses of whether each pedestrian represented a potential collision, accounting for detection failures and braking distance, indicated that pCFL participants were at collision risk for $7 \%$ to $30 \%$ of pedestrians, whereas controls had a rate of 0 to $4 \%$. The reason for the seemingly high hypothetical collisions for the controls is that they often drove faster than the speed limit. Controls were issued "speed warnings" 6 times (0-3 times per participant), whereas only one pCFL participant was given a single speed warning during all sessions.

In summary, despite good visual acuity, and meeting vision requirements for licensing, drivers with para-central scotomas have delayed detection of hazards that fall within the scotomas. Greater account should be taken of both central and para-central scotomas when evaluating vision for driver licensing.

\section{ACKNOWLEDGEMENTS}

Supported in part by NIH grants EY12890 (EP) and EY018680 (ARB). We thank Aaron Mandel for help in scenario development. Christina Gambacorta and Egor Ananev helped with manuscript preparation. Dr. Joseph Rizzo (Boston Veterans Administration Hospital and the Center for Innovative Visual Rehabilitation) provided simulator facilities.

\section{REFERENCES}

Bowers A.R., Mandel A.J., Goldstein R.B., \& Peli E. (2009). Driving with hemianopia, 1: Detection performance in a driving simulator. Investigative Ophthalmology and Visual Science 50, 5137-5147.

Bronstad P.M., Bowers A.R., Goldstein R.B., Albu A., \& Peli E. (2009). The impact of macular degeneration on pedestrian detection: A driving simulator evaluation. Proceedings of the 5th International Driving Symposium on Human Factors in Driver Assessment, Training, and Vehicle Design, Montana, USA. June 22-25. pp. 320-326.

Evans, L. (2004). Traffic Safety. Bloomfield Hills, MI: Science Serving Society.

Fletcher, DC. (2011) Macular Scotoma Interference in Reading - Assessment and Applications. Proceedings of the 10th International Conference on Low Vision, Vision 2011, Kuala Lumpur, Malaysia. CD-ROM Abstract P.2.1

Hassan S.E., Geruschat D.R., \& Turano K.A. (2005). Head movements while crossing streets: Effect of vision impairment. Optometry and Vision Science, 82, 18-26.

Peli E, \& Peli D. Driving with Confidence: A Practical Guide to Driving with Low Vision. Singapore: World Scientific Publishing Co. Pte. Ltd; 2002.

Petzold, A. \& G. T. Plant (2005). "Central and paracentral visual field defects and driving abilities." Ophthalmologica 219, 191-201. 
Pfeiffer, E. (1975). A short portable mental status questionnaire for the assessment of organic brain deficit in elderly patients. Journal of American Geriatrics Society. 23, 433-41.

Rauscher, F. G., C. M. Chisholm, et al. (2007). Central Scotomata and Driving. London, Department of Transport: 1-84.

Turano KA, Geruschat DR, Baker FH. (2002). Fixation behavior while walking: persons with central visual field loss. Vision Research, 42, 2635-2644.

Underwood, G. (2007). Visual attention and the transition from novice to advanced driver. Ergonomics, 50, 1235-1249.

Vargas-Martín F., \& Peli, E. (2006). Eye movements of patients with tunnel vision while walking. Investigative Ophthalmology and Visual Science, 47, 5295-5302.

Woods, R. L., H. L. Apfelbaum, et al. (2010). DLP-based dichoptic vision test system. Journal of Biomedical Optics, 15, 1-13.

Yazdan-Ashoori, P., \& ten Hove, M. (2010). Vision and driving: Canada. Journal of NeuroOphthalmology, 30, 177-185. 